\title{
X-ray Mapping Is 50 Years Young; the Best Is Yet to Come; the Future Is Now!
}

\author{
Dale E. Newbury
}

National Institute of Standards and Technology, Gaithersburg, MD 20899-8370

\section{$\mathrm{X}$-ray mapping is 50 years young:}

X-ray mapping of elemental distributions, first demonstrated by Cosslett and Duncumb 50 years ago, has become one of the most popular modes of operation for exploring chemically heterogeneous microstructures with electron beam microanalysis [1,2]. After 50 years, we might reasonably expect that every aspect of x-ray mapping would have been thoroughly mined for its content. Indeed, all commercial implementations of x-ray mapping for the electron probe microanalyzer (EPMA) and the scanning electron microscope (SEM) include sophisticated software tool kits for x-ray data collection with both wavelength dispersive spectrometry (WDS) and energy dispersive spectrometry (EDS), quantitative processing, and display. EDS data collection can be performed in the x-ray spectrum image (XSI) mode, in which a complete EDS spectrum is recorded at each pixel. The XSI mode captures, in principle, all possible elemental information about the area being scanned within the performance limitations of the spectrometer. The analyst can expect to have the software resources to go beyond simple $\mathrm{x}$-ray mapping with arbitrary scaling of the $\mathrm{x}$ ray intensities in the final display to instead achieve quantitative compositional mapping. Compositional mapping applies all steps followed in conventional fixed-beam quantitative x-ray microanalysis to the single-pixel spectral data: corrections for dose, deadtime, peak overlap and background; standardization and k-value determination (ratio of intensity measured on the unknown to that on the standard); and matrix corrections by the atomic number (Z) - absorption (A) fluorescence (F) method as implemented by conventional ZAF, $\phi(\rho z)$, or "first principles" calculation. In the final display of the compositional map, the gray or color scale is encoded to depict the actual concentration at each pixel, not simply the raw local x-ray intensity, possibly autoscaled without proper concern for the relative elemental efficiency for $\mathrm{x}$-ray generation and measurement. Compositional mapping of minor $(0.01 \leq \mathrm{C} \leq 0.1$ mass fraction $)$ and trace $(\mathrm{C}<0.01)$ constituents avoids the serious artifacts that arise from the composition-dependent $\mathrm{x}$-ray continuum.

\section{The best is yet to come:}

As valuable and popular as x-ray mapping has become, its application has been inevitably limited by two factors: (1) the time penalty for comprehensive compositional mapping imposed by the $\mathrm{x}$ ray ouput count rate (OCR) limitation of conventional EDS (typically about $25 \mathrm{kHz}$ for $\mathrm{Si}(\mathrm{Li}) \mathrm{EDS}$ with a short peaking time appropriate for mapping); and (2) the narrow instantaneous spectral coverage $(\sim 10 \mathrm{eV})$ of WDS, which requires multiple WDS and/or WDS retuning and repeat scans to measure all the elements in a complicated specimen. Until recently, WDS was the only answer when severe peak interference occurred with $\mathrm{Si}(\mathrm{Li})$ EDS or when the limits of detection were required to be below 0.005 mass fraction, which is problematic for EDS. The emergence of the microcalorimeter $\mathrm{x}$-ray spectrometer capable of $4.5 \mathrm{eV}$ resolution at $\mathrm{MnK} \alpha$ with a range of $0.2-10$ $\mathrm{keV}$, and $2 \mathrm{eV}$ resolution at $\mathrm{AlK} \alpha$ for low energy photons $(<2 \mathrm{keV})$, has resulted in EDS with WDS resolution $[3,4,5]$. Existing microcalorimeter EDS detectors have a small solid angle ( $500 \mu \mathrm{sr})$ and are limited to an OCR of $\sim 500-1000 \mathrm{cps}$. Recent research has produced microcalorimeter EDS detector arrays, which can increase the effective solid angle as well as the total output count rate through multiplexing [6]. A 10x10 array could provide an OCR of $50 \mathrm{kHz}-100 \mathrm{kHz}$ with a 
composite resolution, somewhat degraded compared to single detectors, of $15 \mathrm{eV}$ or better. Such a microcalorimeter array could deliver XSI maps with superior resolution and detection sensitivity. The $2 \mathrm{eV}$ resolution achievable at low photon energy enables chemical mapping by observing changes in the shapes and positions of peaks to identify the chemical state of an element.

\section{The future is now:}

The development of digital signal processing (DSP) has improved the OCR performance for mapping with the conventional $\mathrm{Si}(\mathrm{Li})$ EDS to the range $20-50 \mathrm{kHz}$, depending on the resolution (peaking time) acceptable for a specific mapping problem. The recent emergence of the silicon drift detector (SDD) has significantly extended this OCR range [7]. An SDD has been demonstrated to improve the OCR performance by a factor of 5 to 10 for the same resolution compared to $\mathrm{Si}(\mathrm{Li})$ EDS performance [8]. A maximum OCR above $500 \mathrm{kHz}$ has been achieved with a resolution of $217 \mathrm{eV}(\mathrm{MnK} \alpha)$; even higher OCR values may be achieved with multiplexed SDD arrays. Moreover, the SDD can achieve the same resolution with a significantly larger detector area, e.g., a $50 \mathrm{~mm}^{2}$ SDD can achieve $134 \mathrm{eV}(\mathrm{MnK} \alpha)$, which gives greater solid angle and better detection sensitivity per unit radiation dose. This combination of extremely high OCR and large solid angle is ideal for XSI mapping. XSI mapping of minor constituents with $10 \mathrm{~ms}$ dwell per pixel (plus $\sim 1$ ms overhead) and an OCR of $200 \mathrm{kHz}$ has been demonstrated. A 128x128 XSI requires only 3 minutes of accumulation time to detect minor constituents below 0.05 mass fraction [8]. SDD XSI mapping can be performed for major $(\mathrm{C}>0.1$ mass fraction) constituents with a time expenditure similar to that taken to record an SEM backscatter electron (BSE) image with the same pixel density. Moreover, the large databases created in XSI mapping can be efficiently mined for information with various image processing software tools [9, 10]. Such SDD XSI performance brings to the analyst mapping capabilities that until very recently would have been considered "over the rainbow" so that it is not unreasonable to state "the future is now" in x-ray mapping!

\section{References:}

[1] E. Cosslett and P. Duncumb, Nature, 177 (1956) 1172.

[2] J. Goldstein, D. Newbury, D. Joy, C. Lyman, P. Echlin, E. Lifshin, L. Sawyer, and J. Michael, Scanning Electron Microscopy and X-ray Microanalysis, $3^{\text {rd }}$ ed. (New York, Springer, 2003) 485. [3] D. Wollman, K. Irwin, G. Hilton, L. Dulcie, D. Newbury, and J. Martinis, J. Micros., 188 (1987) 196.

[4] K. Irwin, G. Hilton, J. Martinis, S. Deiker, N. Bergren, S. Nam, D. Rudman, and D. Wollman, Nucl. Inst. Meth. Phys Res. A, 444 (2000) 184

[5] D. Wollman, S. Nam, D. Newbury, G. Hilton, K. Irwin, N. Bergren, S. Deiker, D. Rudman, and J. Martinis, Nucl. Instrum. and Methods in Phys. Res. A 444 (2000) 145.

[6] W. Doriese, J. Beall, J. Beyer, S. Deiker, S. Ferreira, G. Hilton, K. Irwin, J. Martinis, S.W. Nam, C. D. Reintsema, J. Ullom, Nucl. Instrum. \& Methods in Phys. Res. A, (2004) 559.

[7] L. Struder, C. Fiorini, E. Gatti, R. Hartmann, P. Holl, N. Krause, P. Lechner, A. Longoni, G. Lutz, J. Kemmer, N. Meidinger, M. Popp, H. Soltau, and C. von Zanthier, Mikrochim. Acta [Suppl] 15 (1998) 11.

[8] D. Newbury SCANNING, 27 (2005) 227.

[9] P. Kotula, M. Keenan and J. Michael, Micros. Microanal. 9 (2003) 1.

[10] D. Newbury and D. Bright, SCANNING, 27 (2005) 15. 